\title{
Heparanase induces a differential loss of heparan sulphate domains in overt diabetic nephropathy
}

\author{
T. J. M. Wijnhoven • M. J. W. van den Hoven • \\ H. Ding • T. H. van Kuppevelt • J. van der Vlag • \\ J. H. M. Berden • R. A. Prinz • E. J. Lewis • \\ M. Schwartz $\cdot$ X. Xu
}

Received: 2 July 2007 / Accepted: 8 October 2007 / Published online: 6 December 2007

(C) Springer-Verlag 2007

\begin{abstract}
Aims/hypothesis Recent studies suggest that loss of heparan sulphate in the glomerular basement membrane (GBM) of the kidney with diabetic nephropathy is due to the increased production of heparanase, a heparan sulphate-degrading endoglycosidase. Our present study addresses whether heparan sulphate with different modifications is differentially reduced in the GBM and whether heparanase selectively cleaves heparan sulphate with different domain specificities.

Methods The heparan sulphate content of renal biopsies (14 diabetic nephropathy, five normal) were analysed by immunofluorescence staining with four anti-heparan sulphate anti-
\end{abstract}

T. J. M. Wijnhoven and M. J. W. van den Hoven contributed equally to this study.

T. J. M. Wijnhoven · T. H. van Kuppevelt

Department of Matrix Biochemistry,

Nijmegen Centre for Molecular Life Sciences,

Radboud University Nijmegen Medical Centre,

Nijmegen, The Netherlands

T. J. M. Wijnhoven

Department of Pediatric Nephrology,

Radboud University Nijmegen Medical Centre,

Nijmegen, The Netherlands

M. J. W. van den Hoven · J. van der Vlag •

J. H. M. Berden

Nephrology Research Laboratory,

Nijmegen Centre for Molecular Life Sciences,

Radboud University Nijmegen Medical Centre,

Nijmegen, The Netherlands

M. J. W. van den Hoven · J. van der Vlag •

J. H. M. Berden

Division of Nephrology,

Radboud University Nijmegen Medical Centre,

Nijmegen, The Netherlands bodies: JM403, a monoclonal antibody (mAb) recognising N-unsubstituted glucosamine residues; two phage displayderived single chain antibodies HS4C3 and EW3D10, defining sulphated heparan sulphate domains; and anti-K5 antibody, an $\mathrm{mAb}$ recognising unmodified heparan sulphate domains.

Results We found that modified heparan sulphate domains (JM403, HS4C3 and EW3D10), but not unmodified domains (anti-K5) and agrin core protein were reduced in the GBM of kidneys from patients with diabetic nephropathy, compared with controls. Glomerular heparanase levels were increased in diabetic nephropathy kidneys and inversely correlated with the amounts of modified heparan sulphate domains. Increased heparanase production and loss of JM403 staining in the GBM

H. Ding $\cdot$ R. A. Prinz $\cdot X . X u(\varangle)$

Department of General Surgery,

Rush University Medical Center,

1653 W. Congress Parkway,

Chicago, IL 60612, USA

e-mail: xxu@rush.edu

\section{E. J. Lewis}

Department of Medicine,

Rush University Medical Centre,

Chicago, IL, USA

M. Schwartz

Department of Pathology,

Rush University Medical Centre,

Chicago, IL, USA 
correlated with the severity of proteinuria. Loss of modified heparan sulphate in the GBM as a result of degradation by heparanase was confirmed by heparan sulphate staining of heparanase-treated normal kidney biopsy specimens.

Conclusions/interpretation Our data suggest that loss of modified heparan sulphate in the GBM is mediated by an increased heparanase presence and may play a role in the pathogenesis of diabetes-induced proteinuria.

Keywords Agrin - Anti-heparan sulphate antibody . Diabetic nephropathy . Glomerular basement membrane . Heparanase $\cdot$ Heparan sulphate $\cdot$ Proteinuria

$\begin{array}{ll}\text { Abbreviations } \\ \text { MAb } & \text { monoclonal antibody } \\ \text { FGF } & \text { fibroblast growth factor } \\ \text { GBM } & \text { glomerular basement membrane } \\ \text { ScFv } & \text { single-chain variable fragment } \\ \text { VEGF } & \text { vascular endothelial growth factor }\end{array}$

\section{Introduction}

Heparan sulphate is a negatively charged glycosaminoglycan linked to a core protein to form a heparan sulphate proteoglycan, which is a major component of extracellular matrices [1]. Due to its negative charge, heparan sulphate in the glomerular basement membrane (GBM) is thought to play an important role in maintaining charge-selective permeability of the glomerular capillary wall by electrostatic repulsion of negatively charged serum proteins, especially albumin. Elimination of GBM heparan sulphate after perfusion of isolated rat kidneys or glomeruli with the bacterial heparan sulphate-degrading enzyme heparinase III resulted in increased permeability of the GBM to native ferritin and albumin [2-4]. Neutralisation of GBM anionic charges after perfusion of isolated rat kidneys or infusion of the renal artery with the cationic probe protamine also resulted in increased permeability of the GBM to albumin $[4,5]$. In addition, binding to GBM heparan sulphate after intravenous injection of rats with the monoclonal anti-heparan sulphate antibody JM403 induced acute selective proteinuria [6].

Patients with diabetes mellitus may develop nephropathy, which is a major cause of end-stage renal disease. Diabetic nephropathy is initially characterised by a minor increase in urinary excretion of albumin, called microalbuminuria, which generally progresses to macroalbuminuria or overt proteinuria with subsequent decline of glomerular function. Ultrastructural changes in diabetic nephropathy include mesangial matrix expansion and thickening of the glomerular and tubular basement membranes [7-9]. In addition, changes in GBM heparan sulphate content have been associated with the pathogenesis of diabetic nephropathy. Studies in patients with overt diabetic nephropathy revealed a decreased heparan sulphate content within the GBM [10-14], which inversely correlated with the degree of albuminuria [11]. Production of the core protein of agrin, the major heparan sulphate proteoglycan in the GBM, was not aberrant $[11,13]$. However, these heparan sulphate changes occur at the macroalbuminuric stage of diabetic nephropathy, since no glomerular heparan sulphate alterations were found at the microalbuminuric stage [15]. Recently, we showed that glomerular production of heparanase, an endo- $\beta$-1,4-D-glucuronidase involved in the hydrolytic cleavage of the $\beta$-1,4-glycosidic bond between glucuronic acid and glucosamine residues within N-sulphated and/or Nacetylated/N-sulphated domains of heparan sulphate $[16,17]$, was increased in patients with overt diabetic nephropathy [11, 18]. In addition, heparanase activity was detected in the urine of diabetic patients [19].

Not only quantitative alterations, but also qualitative differences in GBM heparan sulphate may play a role in the pathogenesis of diabetic nephropathy. Heparan sulphate biosynthesis starts with the addition of a xylose-galactosegalactose-glucuronic acid tetrasaccharide to specific serine residues in the core protein. After addition of the first $\mathrm{N}$ acetylglucosamine, polymerisation takes place by the alternating addition of glucuronic acid and $N$-acetylglucosamine residues. The growing heparan sulphate polysaccharide precursor may undergo extensive but localised modifications, including both $\mathrm{N}$-deacetylation and $\mathrm{N}$-sulphation, $\mathrm{C} 5$ epimerisation and O-sulphation at the $\mathrm{C} 2, \mathrm{C} 6$ and $\mathrm{C} 3$ positions. These modification reactions are generally incomplete, resulting in a high degree of structural diversity in and between heparan sulphate chains. Regions that do not undergo modification remain $\mathrm{N}$-acetylated, regions with intermediate modifications contain alternating $\mathrm{N}$-acetylated and $\mathrm{N}$-sulphated disaccharide units, whereas regions which are highly modified and sulphated consist predominantly of contiguous $\mathrm{N}$ - and $\mathrm{O}$-sulphated disaccharide units.

Although previous studies using JM403, a monoclonal antibody $(\mathrm{mAb})$ that is specific for N-unsubstituted glucosamine residues of heparan sulphate domain, suggest that the GBM of kidneys in patients with diabetic nephropathy has a reduced heparan sulphate content [10-14], it remains to be determined whether loss of heparan sulphate, as detected by JM403, is due to a change of the heparan sulphate structure that favours the binding by this antibody. Alternatively, loss of heparan sulphate in the GBM could be due to the reduced biosynthesis of the nascent heparan sulphate backbone structure. In the present study, we analysed renal heparan sulphate domains using four different anti-heparan sulphate antibodies, directed against sulphated heparan sulphate domains, heparan sulphate domains with $\mathrm{N}$-unsubstituted glucosamine residues or unmodified heparan sulphate 
domains consisting of $\mathrm{N}$-acetylglucosamine-glucuronic acid residues. The experiments were carried out in a group of 13 macroalbuminuric diabetic patients, one microalbuminuric diabetic patient and five control participants, a different group composition from that used in our previous study [11]. Our further studies suggested that heparanase is involved in the degradation of GBM heparan sulphate with both sulphated and $\mathrm{N}$-unsubstituted domains.

\section{Methods}

\section{Participants}

Kidney biopsies from 14 diabetic patients were obtained at the Rush University Medical Center. The biopsies were taken after receiving written informed consent from all participants. At the time of the biopsy, all patients had proteinuria. Characteristics of the patients at the time of the biopsy are summarised in Table 1. Routine histology of the biopsy specimens was conducted to monitor the hyalinisation, Kimmelstiel Wilson nodules, mesangial hypercellularity, interstitial fibrosis and tubular atrophy. Electron microscopy was conducted to monitor the thickness of the GBM and foot process effacement. Control renal tissue was obtained from either non-diabetic donor kidneys that were not suitable for transplantation for anatomical reasons $(n=4)$ or from normal parts of a kidney removed due to renal adenocarcinoma $(n=1)$. The institutional review board of Rush University and the human ethics committee of Radboud University Nijmegen Medical Centre approved all experiments.
Immunofluorescence staining

Two different staining procedures, described below, were used to visualise different heparan sulphate domains, the agrin core protein and heparanase, respectively.

HS4C3 and EW3D10 Frozen kidney sections were air-dried and blocked for $20 \mathrm{~min}$ with PBS containing $2 \%(w / v)$ bovine serum albumin and $0.05 \%(v / v)$ Tween-20 (blocking buffer). Cryosections were then incubated for $1 \mathrm{~h}$ in blocking buffer with the phage display-derived VSV-tagged anti-heparan sulphate antibodies HS4C3 and EW3D10 [20, 21], both of which recognise sulphated heparan sulphate domains. Bound antibodies were detected by incubation with mouse IgG antiVSV tag antibody P5D4 and rabbit IgG anti-VSV tag antibody 9E10 (both 1:10; Boehringer Mannheim, Mannheim, Germany), followed by Alexa 488-conjugated goat antimouse IgG and Alexa 594-conjugated goat anti-rabbit IgG (both 1:200; Molecular Probes, Eugene, OR, USA), respectively, both for $45 \mathrm{~min}$. After each antibody incubation, cryosections were washed with PBS containing $0.1 \%(v / v)$ Tween-20. Finally, cryosections were fixed in $100 \%(v / v)$ ethanol for $\sim 10 \mathrm{~s}$, air-dried and embedded in 10\% (w/v) Mowiol (Calbiochem, La Jolla, CA, USA).

JM403, JM72, anti-K5 antibody and anti-heparanase antibody Frozen kidney sections were air-dried and fixed in $100 \%(v / v)$ acetone for $10 \mathrm{~min}$ at $4^{\circ} \mathrm{C}$. After air-drying, (double) staining was performed by a $1 \mathrm{~h}$ incubation of cryosections with: (1) monoclonal anti-heparan sulphate antibody JM403 (1:300), which recognises heparan sul-

Table 1 Patient characteristics

\begin{tabular}{|c|c|c|c|c|c|c|c|c|}
\hline Patient & Sex & $\begin{array}{l}\text { Age } \\
\text { (years) }\end{array}$ & $\begin{array}{l}\text { Diabetes duration } \\
\text { (years) }\end{array}$ & $\begin{array}{l}\text { GBM width } \\
(\mathrm{nm})\end{array}$ & $\begin{array}{l}\text { Proteinuria } \\
(\mathrm{g} / 24 \mathrm{~h})\end{array}$ & $\begin{array}{l}\text { Serum creatinine } \\
(\mu \mathrm{mol} / 1)\end{array}$ & $\begin{array}{l}\text { Systolic BP } \\
(\mathrm{mmHg})\end{array}$ & $\begin{array}{l}\text { Diastolic BP } \\
(\mathrm{mmHg})\end{array}$ \\
\hline 1 & F & 52 & 15 & N/A & 3.00 & 88 & 133 & 75 \\
\hline 2 & M & 76 & 16 & $>1,000$ & 3.60 & 141 & 163 & 93 \\
\hline 3 & M & 72 & 8 & 400 & 0.13 & 106 & 134 & 59 \\
\hline 4 & M & 74 & 29 & 750 & 1.40 & 177 & 134 & 74 \\
\hline 5 & M & 50 & N/A & 1200 & 7.30 & 495 & 174 & 110 \\
\hline 6 & M & 70 & $>10$ & 500 & 6.30 & 186 & 140 & 60 \\
\hline 7 & $\mathrm{~F}$ & 70 & $>30$ & 1,000 & 1.27 & 80 & 128 & 74 \\
\hline 8 & M & 55 & 15 & 500 & 3.50 & 248 & 136 & 78 \\
\hline 9 & $\mathrm{~F}$ & 59 & N/A & $500-700$ & 3.10 & 274 & 144 & 63 \\
\hline 10 & M & 79 & N/A & $450-625$ & $>5$ & 407 & 118 & 58 \\
\hline 11 & $\mathrm{~F}$ & 68 & $>20$ & $500-800$ & 6.81 & $239-504$ & 213 & 76 \\
\hline 12 & F & 44 & 10 & $<350$ & 4.00 & 88 & 140 & 86 \\
\hline 13 & M & 55 & 15 & $900-1,000$ & 4.50 & 442 & N/A & N/A \\
\hline 14 & F & 36 & N/A & N/A & 2.00 & 362 & N/A & N/A \\
\hline
\end{tabular}

All patients have type 2 diabetes, except for patient 9 (type 1 diabetes)

Macroalbuminuria defined as urinary albumin excretion of $>0.3 \mathrm{~g} / 24 \mathrm{~h}$; serum creatinine normal value: $62-106 \mu \mathrm{mol} / 1$ for men, $44-88 \mu \mathrm{mol} / 1$ for women; systolic BP normal value: $120 \mathrm{mmHg}$; diastolic BP normal value: $80 \mathrm{mmHg}$; GBM width normal value: $300-350 \mathrm{~nm}$

F, female; M, male; N/A, not applicable 
phate domains with $\mathrm{N}$-unsubstituted glucosamine residues $[6,12,22]$ and monoclonal anti-agrin core protein antibody JM72 (1:600) [12, 23]; (2) monoclonal anti-K5 antibody (1:100; Seikagaku, Tokyo, Japan), which recognises unmodified heparan sulphate domains consisting of $N$ acetylglucosamine-glucuronic acid residues; and (3) monoclonal anti-heparanase antibody HP3/17 (1:100; ProsPec-Tany Technogene, Rehovot, Israel) and monoclonal anti-heparan sulphate antibody JM403 (1:300). Bound antibodies were detected by incubation for $1 \mathrm{~h}$ with Alexa 488- or 594conjugated goat anti-mouse IgM (for JM403 and anti-K5 antibody), Alexa 568-conjugated goat anti-mouse IgG1 (for JM72) and Alexa 488-conjugated goat anti-mouse IgG2b (for HP3/17; all 1:200; Molecular Probes) in PBS containing 4\% $(v / v)$ normal human serum and $4 \%(v / v)$ normal goat serum. After each antibody incubation, cryosections were washed with PBS. Finally, cryosections were fixed with $1 \%$ paraformaldehyde $(w / v)$ in PBS for $15 \mathrm{~min}$ and embedded in mounting medium (Vectashield H-1000; Vector Laboratories, Burlingame, CA, USA).

Immunofluorescence staining was evaluated on coded sections by two independent observers using a Leica CTR6000 microscope (Leica Microsystems, Rijswijk, the Netherlands). For JM403, JM72 and anti-K5 antibody, linearity of the GBM was scored on a scale of 0 to $10[0=$ no staining, $5=50 \%$ linear GBM staining, $10=100 \%$ (maximum) GBM staining]. For HS4C3, EW3D10 and anti-heparanase antibody, glomerular staining intensity was scored on a scale of 0 to $5[0=$ no staining, $2.5=50 \%$ staining, $5=100 \%$ (maximum) staining]. Scoring between the different observers was consistent, because regression analysis yielded a good correlation for the scores obtained $(r=0.95, p<0.001)$. The scores given in Figs 1, 2, 3, 4 are the means of all glomeruli per biopsy/kidney.

Fig. 1 Detection of heparan sulphate domains with N-unsubstituted glucosamine residues (JM403) and of the agrin core protein in the GBM of patients with overt diabetic nephropathy and in that of controls. a Immunofluorescence double staining of renal cryosections from a control kidney and a patient with diabetic nephropathy (DNP) using anti-heparan sulphate antibody JM403 (green) and anti-agrin antibody JM72 (red). In control kidneys, a normal linear distribution of heparan sulphate domains recognised by JM403 was detected in the GBM. Heparan sulphate in the GBM co-localised with agrin core protein in controls (merge). However, in patients with diabetic nephropathy, we observed a decreased presence in the GBM of heparan sulphate domains recognised by JM403, whereas agrin levels were unaltered. b Quantification of the staining revealed that amounts of heparan sulphate domains with $\mathrm{N}$-unsubstituted glucosamine residues detected with JM403 were significantly reduced in the GBM of patients with diabetic nephropathy compared with that of controls. ${ }^{*} p<0.01$ c No differences in agrin core protein levels were observed between controls and diabetic patients. d An inverse correlation between amounts of heparan sulphate domains recognised by JM403 and the level of proteinuria was observed. $r=-0.56$; $p<$ 0.05 . Because of the lack of glomeruli in one biopsy, only 13 biopsies were evaluated. AU, arbitrary units
Immunohistochemical staining

Heparanase localisation was also visualised by immunohistochemical staining. Paraffin-embedded tissue sections were de-waxed with xylene and rehydrated. Endogenous
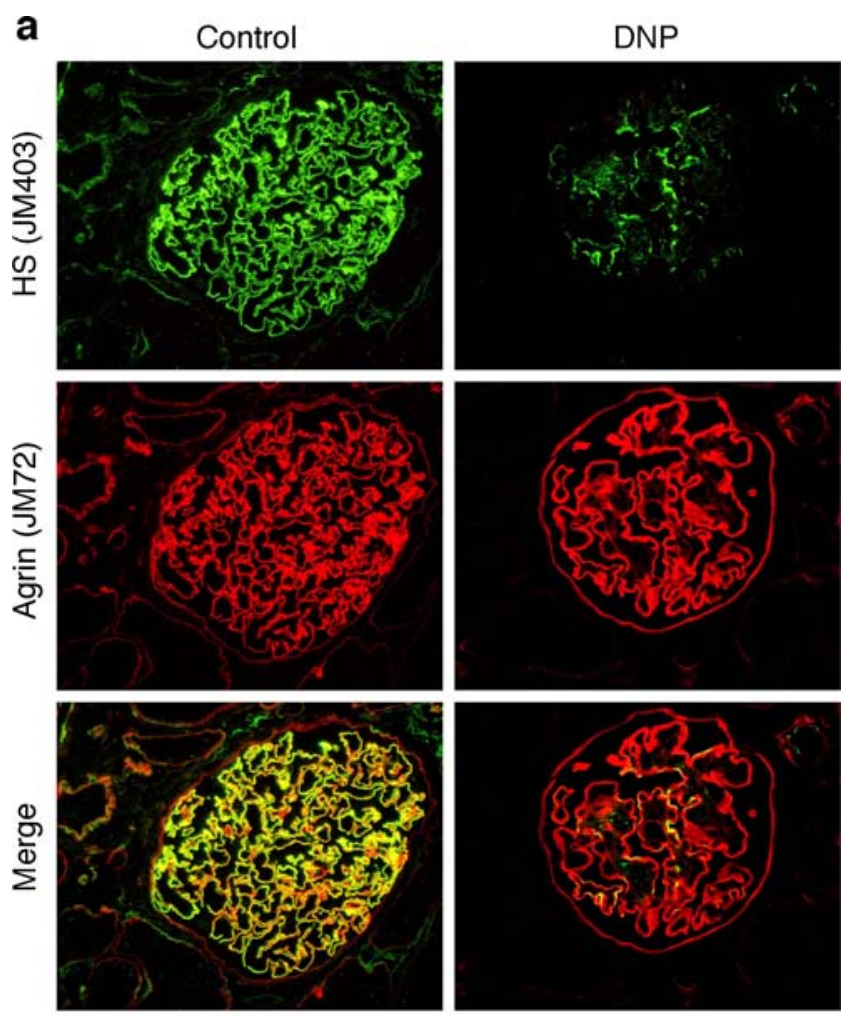

b

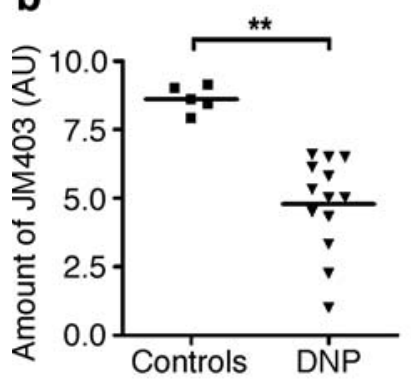

C

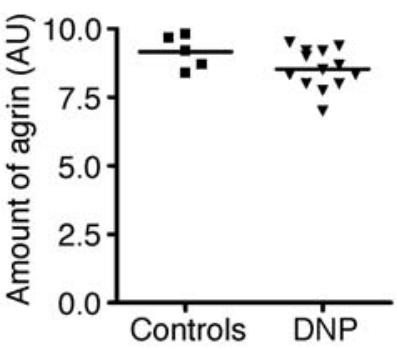

d

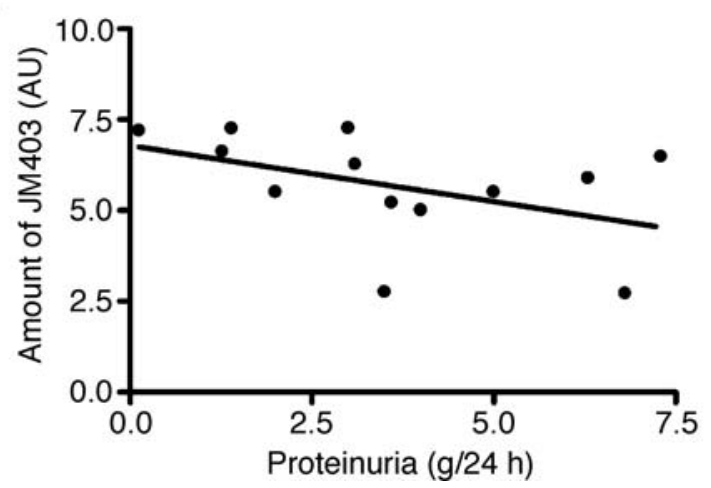



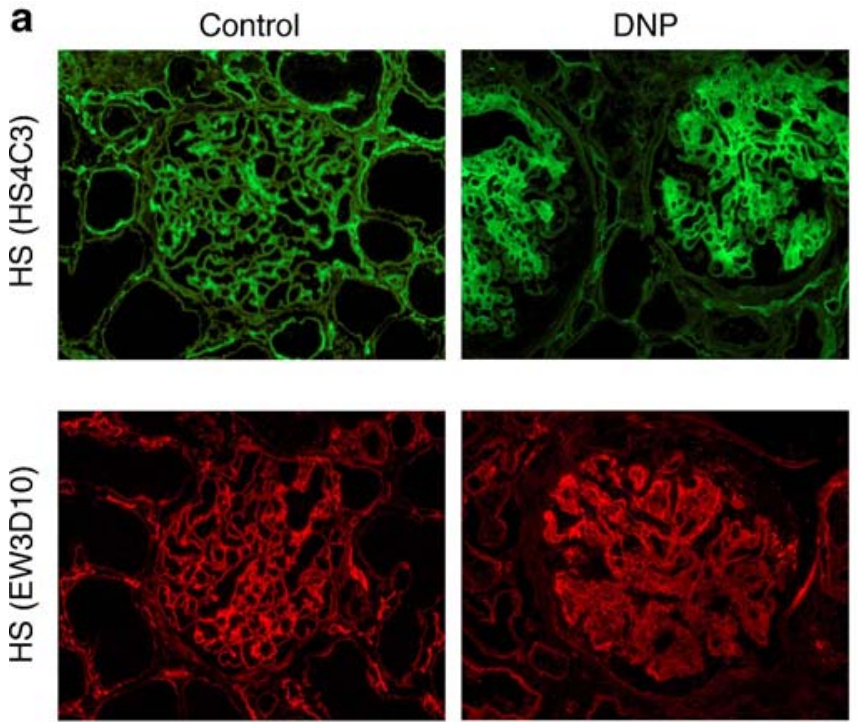

Fig. 2 Detection of sulphated heparan sulphate domains (HS4C3 and EW3D10) in the GBM and mesangium of patients with overt diabetic nephropathy and of controls. a Immunofluorescence staining of renal cryosections from a control kidney and a patient with diabetic nephropathy (DNP) using anti-heparan sulphate antibody HS4C3 (green) and anti-heparan sulphate antibody EW3D10 (red), both of which predominantly recognise sulphated heparan sulphate domains in the mesangium, but also recognise sulphated heparan sulphate domains in the GBM. Amounts of heparan sulphate domains recognised by $\mathrm{HS} 4 \mathrm{C} 3$ and $\mathrm{EW} 3 \mathrm{D} 10$ were reduced in the GBM of

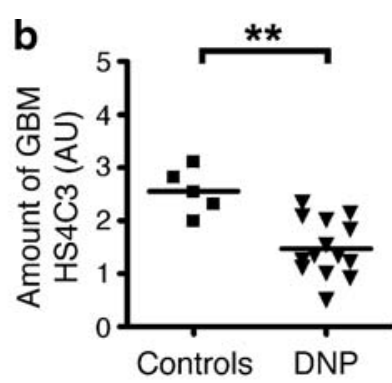

d
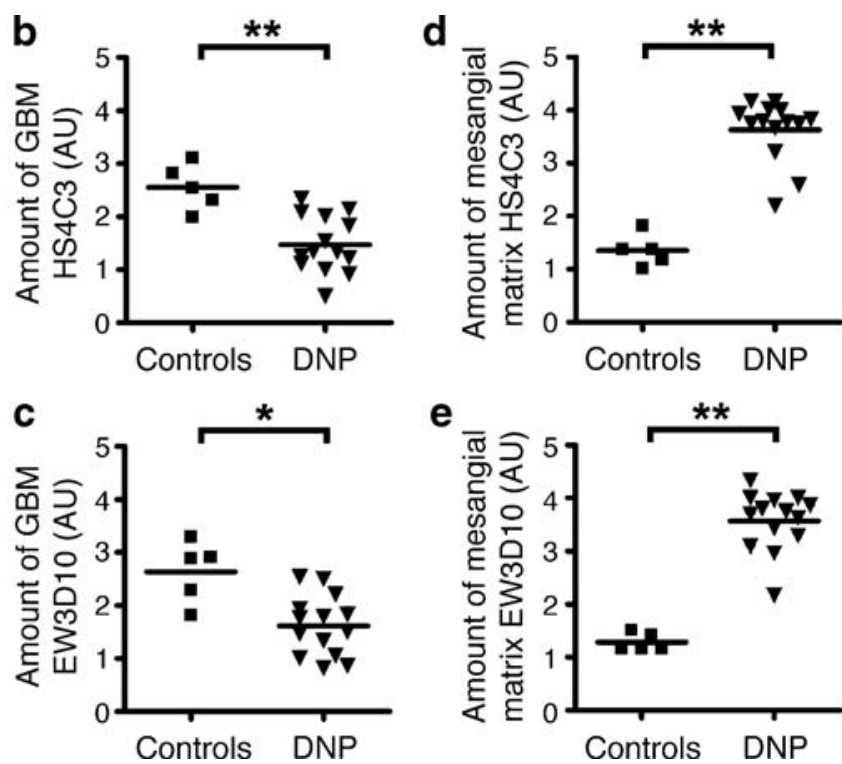

e

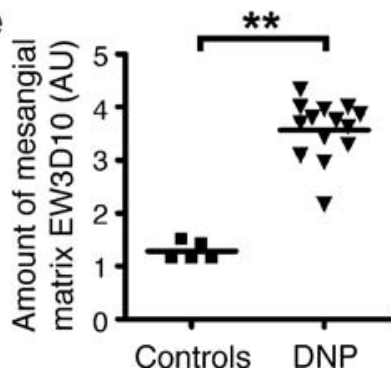

diabetic patients compared with controls. The staining intensity of these antibodies in the mesangium was comparable in patients and controls, whereas in the patients with diabetic nephropathy mesangial matrix expansion was observed. b-e Quantification analysis of the staining revealed that amounts of sulphated heparan sulphate domains recognised by HS4C3 (b) and EW3D10 (c) in the GBM of patients with diabetic nephropathy were significantly reduced. Staining with HS4C3 (d) and EW3D10 (e) revealed a significantly increased mesangial matrix expansion in patients with diabetic nephropathy. $* p<0.05, * * p<0.01$. AU, arbitrary units

peroxidases were inactivated in 3\% $(v / v)$ hydrogen peroxide for $10 \mathrm{~min}$. Sections were processed for immunohistochemical staining with a rabbit anti-heparanase antiserum (anti-HPR1) as previously described [18]. The same concentration of a normal rabbit serum was used as a negative control.

Treatment of renal sections with active heparanase

Cryosections of normal human kidneys were treated for $2 \mathrm{~h}$ with $0.5 \mu \mathrm{g} / \mathrm{ml}$ active human recombinant heparanase in $50 \mathrm{mmol} / 1$ sodium acetate buffer $(\mathrm{pH} 5.0)$ at $37^{\circ} \mathrm{C}$. After washing the sections in PBS, staining for different heparan sulphate domains (using JM403, HS4C3, EW3D10 and anti-K5 antibody) and the agrin core protein (using JM72) was performed as described above.

\section{Statistical analysis}

Differences between groups were determined by the nonparametric Mann-Whitney $U$ test using GraphPad Prism 4.0 (GraphPad Software, San Diego, CA, USA). The nonparametric Spearman's rank test was used to calculate correlation between different variables. Statistical significance was regarded if $p<0.05$.

\section{Results}

Differential loss of heparan sulphate domains in the glomerular basement membrane of patients with overt diabetic nephropathy

Our recent study showed a reverse correlation between the content of the GBM heparan sulphate and proteinuria [11]. Here we attempted to see whether these observations could be confirmed in a different patient population. The distribution of heparan sulphate in the GBM and mesangium was evaluated by immunofluorescence (double) staining of kidney biopsies from proteinuric diabetic patients and normal kidneys. Control kidney sections showed a linear distribution of the agrin core protein along the GBM (Fig. 1a) and heparan sulphate staining, using antibody JM403, co-localised with the agrin staining (Fig. 1a). However, glomeruli of patients with diabetic nephropathy showed a decreased presence of heparan sulphate domains with $\mathrm{N}$-unsubstituted glucosamine residues along the GBM, whereas the amount of agrin remained unaltered. Quantitative analysis revealed that the amount of heparan sulphate domains in the GBM recognised by JM403 was significantly decreased in diabetic nephropathy patients (Fig. 1b), whereas the amount of the 

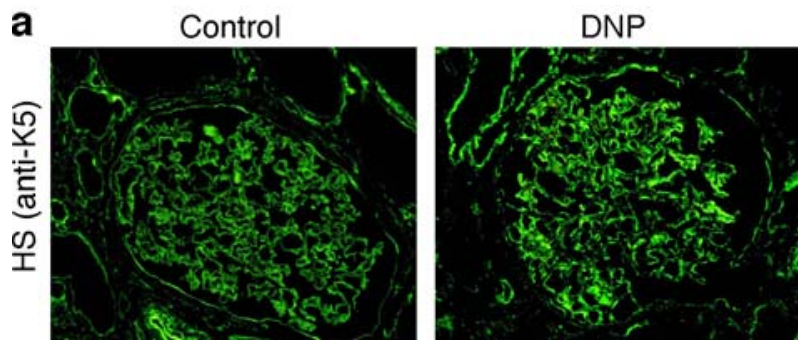

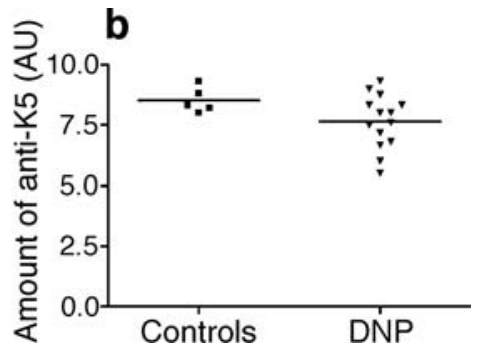

Fig. 3 Glomerular distribution of unmodified heparan sulphate domains (anti-K5 antibody) in the GBM of patients with overt diabetic nephropathy and of controls. a Immunofluorescence staining of renal cryosections from a control kidney and a patient with diabetic nephropathy using anti-K5 antibody, which recognises unmodified heparan sulphate domains consisting of $\mathrm{N}$-acetylglucosamine-glucuronic acid residues (green). No differences in amounts of unmodified heparan sulphate domains in the GBM were observed between the controls and the diabetic patients. b Quantification of the staining revealed no significant differences in levels of unmodified heparan sulphate domains between diabetic patients and the controls. AU, arbitrary units agrin core protein was not different (Fig. 1c). These results indicate that the decreased staining of the GBM by antiheparan sulphate antibody JM403 is not due to reduced synthesis of the core protein of agrin, which is the major heparan sulphate proteoglycan in the GBM [24, 25]. In this analysis we also observed an inverse correlation between the heparan sulphate domain content of the GBM and the level of proteinuria (Fig. 1d). However, we did not find any correlation between the GBM width (Table 1) and heparan sulphate signal intensity, suggesting that reduced heparan sulphate in the GBM of diabetic nephropathy kidneys is not due to the diffused presence of heparan sulphate resulting from expansion of the GBM. There was also no correlation between loss of the GBM heparan sulphate and foot process effacement or the severity of arteriole sclerosis and hyalinisation (data not shown).

In addition to heparan sulphate domains containing $\mathrm{N}$ unsubstituted glucosamine residues, the presence of sulphated heparan sulphate domains was studied using the phage display-derived anti-heparan sulphate antibodies HS4C3 and EW3D10. These antibodies predominantly stained the mesangial matrix, but also the GBM in control kidneys (Fig. 2a). Analysis revealed that the amounts of sulphated heparan sulphate domains in the GBM, as detected with HS4C3 and EW3D10, were significantly decreased in patients with diabetic nephropathy (Fig. 2b), whereas the staining intensity of the mesangial matrix was not altered (data not shown). However, using both antibodies, we observed obvious mesangial matrix expansion in the diabetic patients (Fig. 2c). Interestingly, staining for the unmodified heparan sulphate domains with $\mathrm{N}$-acetylglucosamineglucuronic acid residues, i.e. heparan sulphate precursor domain, using anti-K5 antibody led to similar results in controls and diabetic nephropathy patients (Fig. 3a,b). This latter result suggests that either remnants of heparan sulphate or non-modified heparan sulphate side chains are still present in the GBM.
Increased heparanase content in the glomeruli of patients with overt diabetic nephropathy

Emerging evidence suggests that heparanase production is increased in glomerular epithelial cells (podocytes) and glomerular endothelial cells of patients with diabetic

a
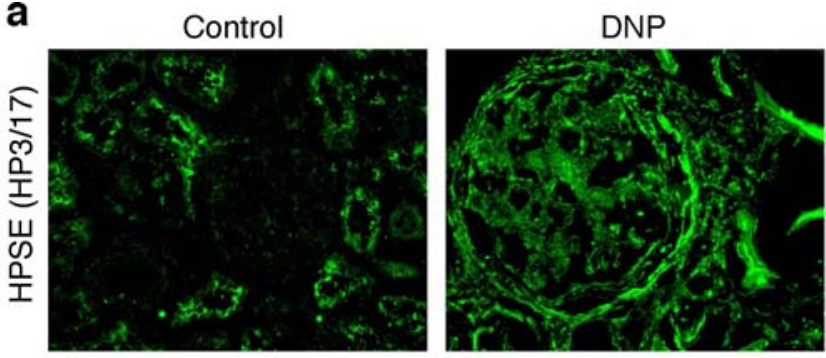

b

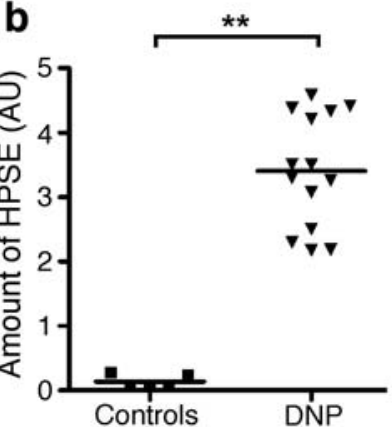

C

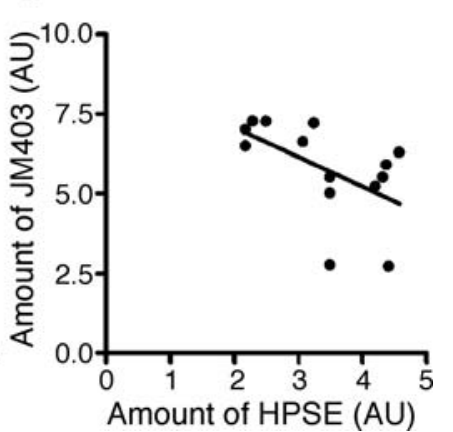

Fig. 4 Analysis of glomerular heparanase in patients with overt diabetic nephropathy and in controls. a Immunofluorescence staining of renal cryosections from a control kidney and a patient with diabetic nephropathy (DNP) using anti-heparanase antibody HP3/17 (green) revealed increased glomerular amounts of heparanase (HPSE) in patients with diabetic nephropathy. b Quantification of the staining revealed that patients with diabetic nephropathy had significantly increased glomerular heparanase levels compared with controls. $* * p<$ 0.01. c Reduced amounts of heparan sulphate domains in the GBM, as detected with anti-heparan sulphate antibody JM403, correlated with increased levels of heparanase. $r=-0.63 ;{ }^{*} p<0.05$. AU, arbitrary units 
nephropathy $[11,18]$, as well as in glomerular cells cultured under high glucose conditions [18]. Here we tested whether loss of GBM heparan sulphate may be due to increased heparanase production. Heparanase signals were very weak in normal human glomeruli, but increased in glomeruli of diabetic nephropathy patients (Fig. 4a). The increased presence of glomerular heparanase in patients with diabetic nephropathy compared with controls was statistically significant (Fig. 4b). Analysis of the immunofluorencence doublestaining with JM403 and heparanase revealed that the decreased presence of heparan sulphate domains with $\mathrm{N}$-unsubstituted glucosamine residues in the GBM correlated with increased glomerular heparanase levels (Fig. 4c). These data suggest that heparanase synthesised in the glomeruli of patients with overt diabetic nephropathy plays an important role in the degradation of heparan sulphate in the GBM. However, no correlation was seen between heparanase content or heparan sulphate loss and mesangial matrix expansion or GBM thickness (data not shown).

Since immunofluorescence staining does not allow a proper localisation of heparanase, we also performed in situ immunohistochemistry with a different antibody that can be used on paraffin-embedded sections [18]. As shown in Fig. 5a and b, strong heparanase signals were present in the podocytes (red arrows) and in the proximal tubular cells (green arrows) in a specimen from a patient with overt diabetic nephropathy. Again, heparanase was not present in the glomerulus and only weakly stained in the tubules of a normal kidney.
Loss of heparan sulphate domains in the glomerular basement membrane is mediated by heparanase

We reasoned that if the loss of heparan sulphate domains in the GBM of diabetic patients was due to the degradation of heparan sulphate by heparanase, incubation of normal renal cryosections with active heparanase should lead to similar data to those obtained for patients with diabetic nephropathy, i.e. differential loss of heparan sulphate domains. Indeed, treatment of normal human kidney cryosections with active heparanase did result in a complete loss of the heparan sulphate domains recognised by antibodies JM403 and EW3D10, while presence of the heparan sulphate domain recognised by antibody HS4C3 was strongly decreased. The renal content of unmodified heparan sulphate domains consisting of $\mathrm{N}$-acetylglucosamine-glucuronic acid and of the agrin core protein was not affected by heparanase treatment (Fig. 6). These data mirror the staining observed in the diabetic nephropathy patients, strongly suggesting that increased glomerular production of heparanase is responsible for the differential loss of heparan sulphate domains in the GBM of patients with overt diabetic nephropathy.

\section{Discussion}

Our study shows that levels of the agrin core protein in the GBM in overt diabetic nephropathy were similar to those in controls, whereas modified heparan sulphate domains with $\mathrm{N}$ -
Fig. 5 Renal localisation of heparanase in patients with overt diabetic nephropathy. a Immunohistochemical staining of paraffin-embedded renal sections from a patient with diabetic nephropathy using a rabbit anti-heparanase antiserum (antiHPR1) revealed strong heparanase signals in the podocytes (red arrows) and in the proximal tubular cells (green arrows). b Enlargement of blue-framed inset in (a). A normal kidney specimen (c) was included and showed no heparanase signal in the glomerulus and weak signals in the tubule. $\mathbf{d}$ A normal rabbit serum for a biopsy specimen was included as negative control

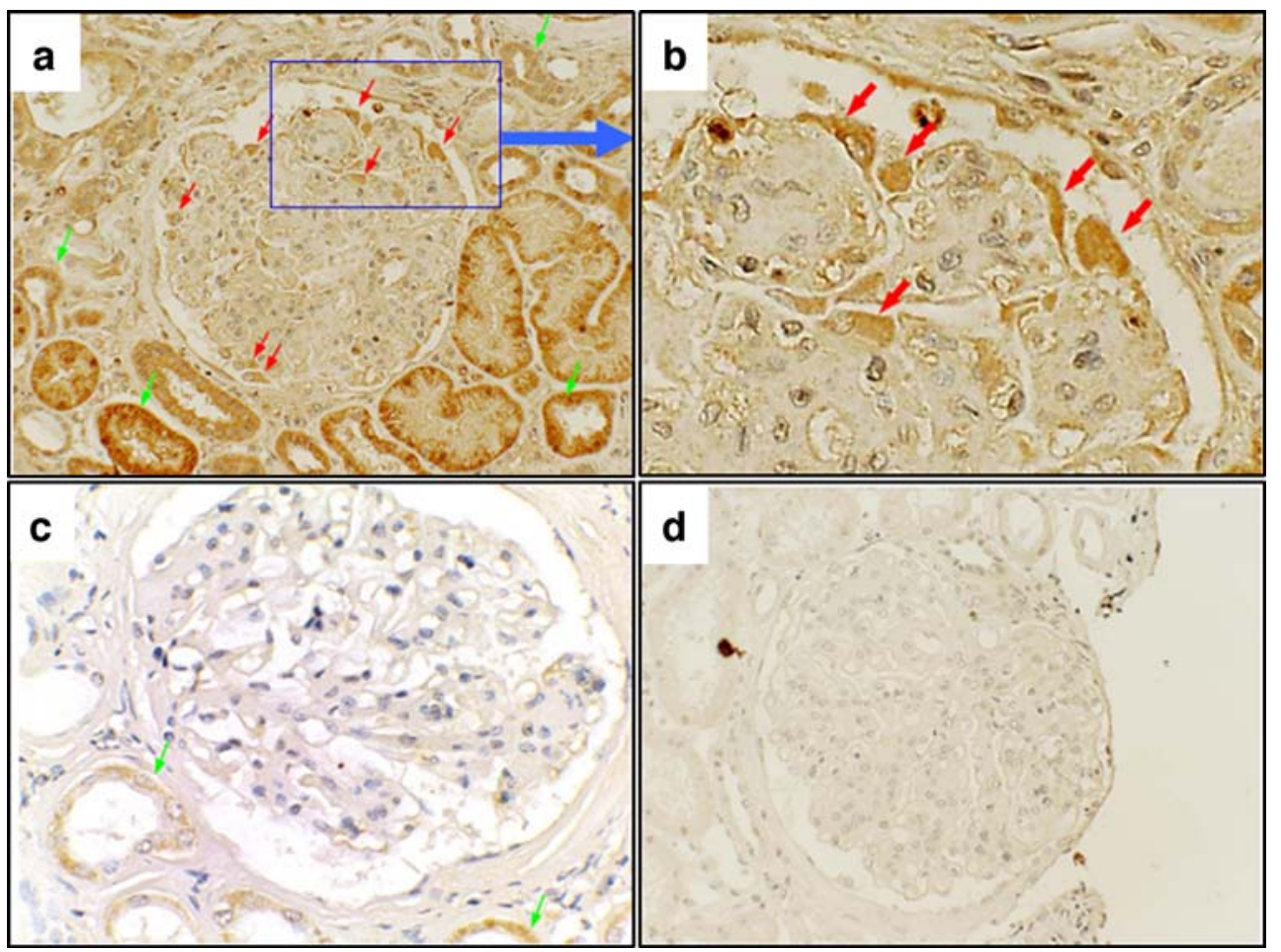



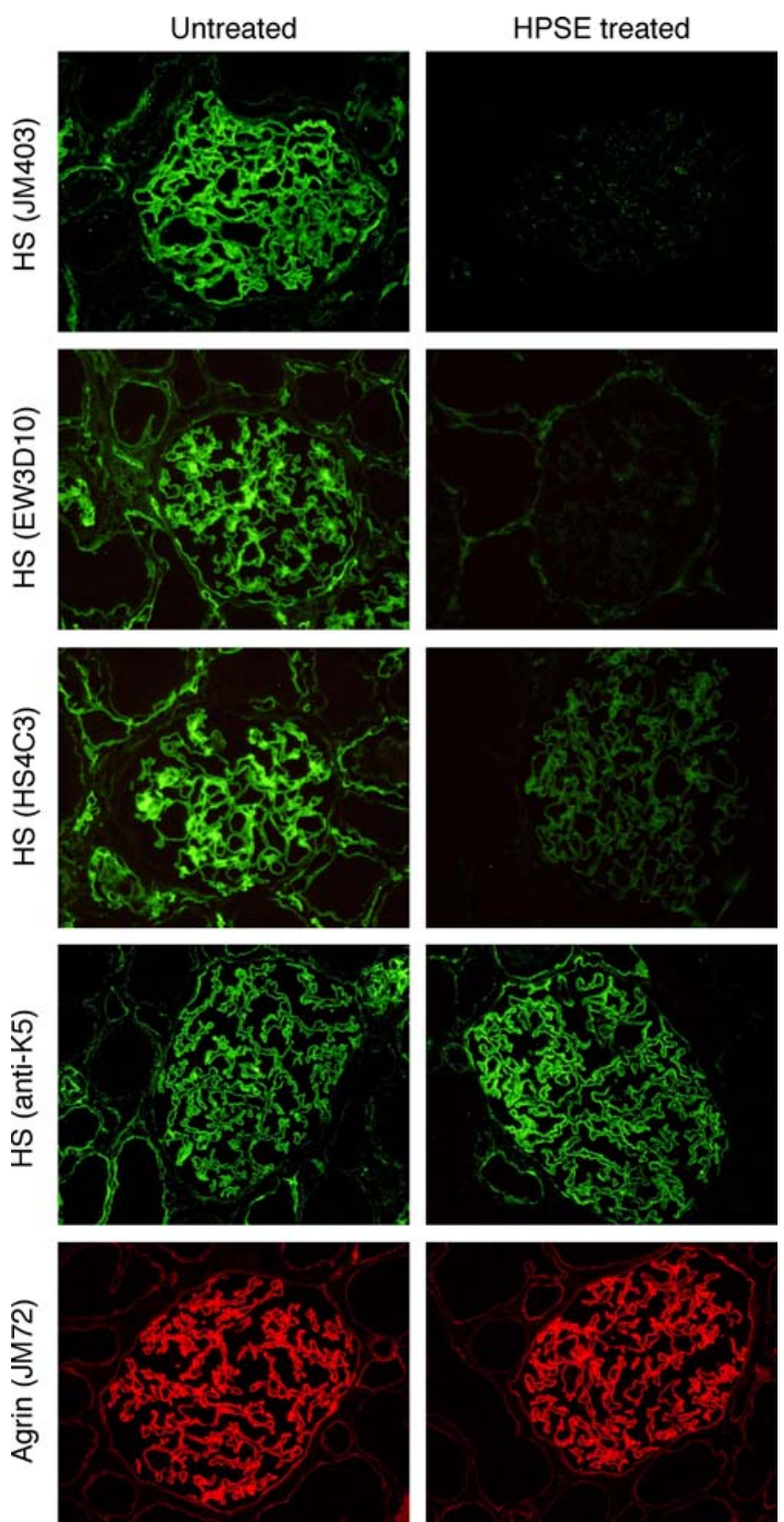

Fig. 6 Detection of different heparan sulphate domains and of the agrin core protein after treatment of normal human kidney sections with active heparanase. After treatment of normal human kidney cryosections with active recombinant heparanase, heparan sulphate domains recognised by JM403 and EW3D10 completely disappeared, whereas heparan sulphate domains recognised by HS4C3 were only strongly reduced. In contrast, unmodified heparan sulphate domains detected with anti-K5 and the agrin core protein were not affected by heparanase treatment

unsubstituted glucosamine residues (detected with JM403 antibody) and sulphated heparan sulphate domains [detected with two phage display-derived single-chain variable fragment ( $\mathrm{scFv}$ ) antibodies] were undetectable or greatly reduced in overt diabetic nephropathy. In contrast, unmodified heparan sulphate domains, detected with anti-K5 mAb and consisting of $\mathrm{N}$-acetylglucosamine-glucuronic acid residues, remained unchanged. Since heparanase preferentially cleaves sulphated heparan sulphate and the glomerular heparanase content was increased in patients with overt diabetic nephropathy and inversely correlated with levels of modified heparan sulphate in the GBM, it is conceivable that the reduction of modified heparan sulphate in the GBM was due to degradation by heparanase, whereas the unmodified heparan sulphate domains, which are resistant to heparanase degradation [26], may represent the nascent chains of heparan sulphate or the large fragments of sparsely sulphated heparan sulphate after heparanase degradation. In support of this notion, pretreatment of normal human kidney sections with the active recombinant human heparanase did not reduce the presence of unmodified heparan sulphate (detected by anti$\mathrm{K} 5 \mathrm{mAb}$ ), whereas a complete loss or significant reduction of modified heparan sulphate domains (detected with antibodies JM403 and EW3D10 or HS4C3 respectively) was seen. Consistent with these studies, we have also found that only the unmodified heparan sulphate domain can be detected by the anti-K5 antibody in the GBM of transgenic mice overexpressing heparanase [27]. Taken together, our observations strongly suggest that agrin gene expression and biosynthesis of the heparan sulphate precursor are not reduced in human diabetic nephropathy and that the reduction of modified heparan sulphate domains with $\mathrm{N}$-unsubstituted glucosamine residues and sulphated heparan sulphate domains is due to accelerated degradation by increased heparanase production.

While our study suggests that heparanase plays an important role in eliminating modified heparan sulphate in the GBM of patients with overt diabetic nephropathy, we surprisingly found that the presence of sulphated heparan sulphate, as detected by the two $\mathrm{scFv}$ antibodies, was not decreased in the mesangium of kidneys with overt diabetic nephropathy. There are several putative explanations for this paradox. First, our recent study demonstrated that heparanase was largely located in the GBM and podocytes, but not in the mesangial cells [11]. Therefore, an increased heparan sulphate content in the mesangium of kidneys with overt diabetic nephropathy could be due to mesangial matrix expansion and the lack of heparanase degradation. Second, heparan sulphate-binding growth factors such as fibroblast growth factor (FGF) and vascular endothelial growth factor (VEGF), which are abundantly present in the mesangium $[28,29]$, may bind and protect heparan sulphate from heparanase access and cleavage. Third, increased heparan sulphate in the mesangium may be due to special modifications of heparan sulphate domains that are preferentially recognised by the two $\mathrm{scFv}$ antibodies. Nevertheless, it appears that lack of heparan sulphate reduction in the mesangium is pathologically relevant, since heparan sulphate in the mesangium serves as the co-receptor for many growth factors such as FGF and VEGF $[28,29]$ and 
can enhance their mitogenic effect on mesangial cells, leading to increased cell proliferation, extracellular matrix protein production and mesangial matrix expansion.

In the past 5 years, upregulation of glomerular heparanase gene expression has been shown in numerous clinical or experimental nephropathies such as puromycin aminonucleoside nephrosis [30], passive Heymann nephritis [31], anti-GBM nephritis [32], adriamycin nephropathy [33], streptozotocin-induced diabetic nephropathy [11], minimal change nephrotic syndrome, and membranous glomerulopathy [34]. The molecular mechanisms that regulate heparanase gene expression and activity still have to be elucidated. Preliminary evidence from our laboratories suggests that hyperglycaemia, angiotensin II, aldosterone, reactive oxygen species and activation of B-Raf kinase in different cell types are involved in regulating the production of heparanase. Heparanase production was increased under high glucose conditions in cultured podocytes, the main cell type producing heparanase [18]. Very recently, we demonstrated increased heparanase gene expression in adriamycin nephropathy, which was reduced or prevented after treatment with an angiotensin II receptor antagonist or a scavenger of hydroxyl radicals [33]. We also have preliminary data that aldosterone may regulate heparanase production, since we observed increased heparanase gene expression after stimulation of podocytes with aldosterone. This effect could be abrogated by pretreatment with an aldosterone receptor blocker (M. van den Hoven, J. van der Vlag and J. Berden, unpublished results).

It has long been postulated that heparan sulphate plays an important role in the charge-selective permeability properties of the GBM and the development of proteinuria. For example, rats developed massive albuminuria after injection of the mAb JM403 [6]. Degradation of heparan sulphate by the bacterial heparan sulphate-cleaving enzyme heparinase III resulted in an increased permeability of the GBM to ferritin and albumin [2-4]. In addition, a loss of heparan sulphate in the GBM has been observed in many glomerular diseases, including diabetic nephropathy, and correlated with the degree of proteinuria [10-14]. However, several recent studies suggest that heparan sulphate in the GBM may not play a primary decisive role in the chargeselective permeability properties and development of proteinuria. For example, van den Born recently reported that there was no change in glomerular heparan sulphate content in early human and experimental diabetic nephropathy, although in both cases albuminuria was present [15]. Additional studies have shown that rats did not develop acute albuminuria after in vivo degradation of heparan sulphate by heparinase III [35]. Heparanase transgenic mice with complete loss of glomerular heparan sulphate only developed very mild albuminuria [11]. Furthermore, podocyte-specific agrin knockout mice [36] and podocyte- specific exostosis-1 (EXT1) knockout mice [37] have been generated, both of which lack heparan sulphate in the GBM. Despite the absence of heparan sulphate, these mice do not develop proteinuria [36] either under normal conditions or when they are overloaded with albumin. While these recent data suggest that loss of heparan sulphate in the GBM alone does not lead to proteinuria, it is not clear whether loss of heparan sulphate under pathological conditions such as diabetic nephropathy accelerates or worsens proteinuria. An earlier study showed that loss of another heparan sulphate proteoglycan, perlecan, leads to proteinuria in albumin-overloaded perlecandeficient mice [38]. We speculate that loss of heparan sulphate may contribute to the pathogenesis of proteinuria under prolonged hyperglycaemic conditions. Our present study, along with a recent related study by our group [11] demonstrating that the reduction of glomerular heparan sulphate correlated with the degree of proteinuria, supports this notion. Loss of heparan sulphate in the GBM may exacerbate proteinuria not by directly increasing the permeability of the GBM, but rather by other indirect mechanisms, such as affecting the mitogenic effect of heparan sulphatebinding growth factors (FGF-2, VEGF, TGF- $\beta$, connective tissue growth factor) and disrupting the cell-GBM interactions, leading to dedifferentiation of glomerular cells and loss of podocytes. Loss of heparan sulphate may also result in structural GBM alterations and compromise the integrity of the glomerular capillary wall. Finally, loss of heparan sulphate in the GBM may increase the deposition of lipoproteins. Nevertheless, use of heparanase knockout mice or conditioned heparanase knockout mice and heparanasespecific inhibitors will provide direct and definitive evidence to clarify whether heparanase is a critical molecule for the development of proteinuria.

The supposition that loss of heparan sulphate in the GBM contributes to proteinuria in overt diabetic nephropathy is indirectly supported by the observation of an antiproteinuric effect in diabetic patients treated with several glycosaminoglycan-based drugs, including heparin (highly sulphated heparan sulphate) [39], sulodexide (80\% fast-moving heparin and 20\% dermatan sulphate) [40-43], enoxaparin (low molecular weight heparin) [44] and danaparoid (mixture of glycosaminoglycans consisting predominantly of heparan sulphate) [45]. Since these drugs can also inhibit heparanase activity [46, 47], it is possible that their ameliorating effect on proteinuria is due to inhibition of heparanase. A study by Levidiotis et al. [48] showed that inhibition of heparanase with the specific heparanase inhibitor PI- 88 reduced proteinuria in passive Heymann nephritis.

In summary, our present study showed that amounts of modified heparan sulphate domains were reduced in the GBM of patients with overt diabetic nephropathy, whereas 
amounts of the unmodified precursor of heparan sulphate were unaltered. The loss of heparan sulphate in the GBM correlated with an increased presence of glomerular heparanase in these patients. In addition, the heparan sulphate content also correlated with the degree of proteinuria. Our data suggest that the increased glomerular heparanase production in patients with diabetic nephropathy may be responsible for the differential loss of heparan sulphate domains in the GBM, which may participate in the pathogenesis of diabetes-induced proteinuria.

Acknowledgements This study was supported by grants from the Netherlands Organization for Scientific Research (NWO) (programme grant 902-27-292), the Dutch Diabetes Research Foundation (grant 2001.00.048) and Rush University Research Council. We thank I. Vlodavsky (Cancer and Vascular Biology Research Center, Rappaport Faculty of Medicine, Technion, Haifa, Israel) for the generous gift of human recombinant heparanase and R. L. Heinrikson (Pharmacia \& Upjohn, Kalamazoo, MI, USA) for kindly providing anti-heparanase rabbit antiserum.

Duality of interest The authors declare that there is no duality of interest associated with this manuscript.

\section{References}

1. Esko JD, Selleck SB (2002) Order out of chaos: assembly of ligand binding sites in heparan sulfate. Annu Rev Biochem 71:435-471

2. Kanwar YS, Linker A, Farquhar MG (1980) Increased permeability of the glomerular basement membrane to ferritin after removal of glycosaminoglycans (heparan sulfate) by enzyme digestion. J Cell Biol 86:688-693

3. Kanwar YS, Rosenzweig LJ, Kerjaschki DI (1981) Glycosaminoglycans of the glomerular basement membrane in normal and nephrotic states. Ren Physiol 4:121-130

4. Daniels BS (1994) Increased albumin permeability in vitro following alterations of glomerular charge is mediated by the cells of the filtration barrier. J Lab Clin Med 124:224-230

5. Vehaskari VM, Root ER, Germuth FG, Jr, Robson AM (1982) Glomerular charge and urinary protein excretion: effects of systemic and intrarenal polycation infusion in the rat. Kidney Int 22:127-135

6. van den Born J, van den Heuvel LP, Bakker MA, Veerkamp JH, Assmann KJ, Berden JH (1992) A monoclonal antibody against GBM heparan sulfate induces an acute selective proteinuria in rats. Kidney Int 41:115-123

7. Mauer SM, Steffes MW, Ellis EN, Sutherland DE, Brown DM, Goetz FC (1984) Structural-functional relationships in diabetic nephropathy. J Clin Invest 74:1143-1155

8. Ibrahim HA, Vora JP (1999) Diabetic nephropathy. Baillieres Best Pract Res Clin Endocrinol Metab 13:239-264

9. Danis D, Nyitrayova O, Slugen I, Kovac A, Nyulassy S, Orban A (1996) Tubular basement membrane thickening in diabetes mellitus. Int Urol Nephrol 28:589-592

10. Makino H, Yamasaki Y, Haramoto T et al (1993) Ultrastructural changes of extracellular matrices in diabetic nephropathy revealed by high resolution scanning and immunoelectron microscopy. Lab Invest 68:45-55

11. van den Hoven MJ, Rops AL, Bakker MA et al (2006) Increased expression of heparanase in overt diabetic nephropathy. Kidney Int 70:2100-2108
12. van den Born J, van den Heuvel LP, Bakker MA et al (1993) Distribution of GBM heparan sulfate proteoglycan core protein and side chains in human glomerular diseases. Kidney Int 43: 454-463

13. Tamsma JT, van den Born J, Bruijn JA et al (1994) Expression of glomerular extracellular matrix components in human diabetic nephropathy: decrease of heparan sulphate in the glomerular basement membrane. Diabetologia 37:313-320

14. Deckert T, Feldt-Rasmussen B, Djurup R, Deckert M (1988) Glomerular size and charge selectivity in insulin-dependent diabetes mellitus. Kidney Int 33:100-106

15. van den Born J, Pisa B, Bakker MA et al (2006) No change in glomerular heparan sulfate structure in early human and experimental diabetic nephropathy. J Biol Chem 281:29606-29613

16. Bame KJ (2001) Heparanases: endoglycosidases that degrade heparan sulfate proteoglycans. Glycobiology 11:91R-98R

17. Vlodavsky I, Friedmann Y (2001) Molecular properties and involvement of heparanase in cancer metastasis and angiogenesis. J Clin Invest 108:341-347

18. Maxhimer JB, Somenek M, Rao G et al (2005) Heparanase-1 gene expression and regulation by high glucose in renal epithelial cells: a potential role in the pathogenesis of proteinuria in diabetic patients. Diabetes 54:2172-2178

19. Katz A, Van-Dijk DJ, Aingorn H et al (2002) Involvement of human heparanase in the pathogenesis of diabetic nephropathy. Isr Med Assoc J 4:996-1002

20. van Kuppevelt TH, Dennissen MA, van Venrooij WJ, Hoet RM, Veerkamp JH (1998) Generation and application of type-specific anti-heparan sulfate antibodies using phage display technology. Further evidence for heparan sulfate heterogeneity in the kidney. J Biol Chem 273:12960-12966

21. van de Westerlo EM, Smetsers TF, Dennissen MA et al (2002) Human single chain antibodies against heparin: selection, characterization, and effect on coagulation. Blood 99:2427-2433

22. van den Born J, Gunnarsson K, Bakker MA et al (1995) Presence of $\mathrm{N}$-unsubstituted glucosamine units in native heparan sulfate revealed by a monoclonal antibody. J Biol Chem 270:31303-31309

23. van den Born J, van den Heuvel LP, Bakker MA, Veerkamp JH, Assmann KJ, Berden JH (1994) Monoclonal antibodies against the protein core and glycosaminoglycan side chain of glomerular basement membrane heparan sulfate proteoglycan: characterization and immunohistological application in human tissues. J Histochem Cytochem 42:89-102

24. Groffen AJ, Ruegg MA, Dijkman H et al (1998) Agrin is a major heparan sulfate proteoglycan in the human glomerular basement membrane. J Histochem Cytochem 46:19-27

25. Raats CJ, Bakker MA, Hoch W et al (1998) Differential expression of agrin in renal basement membranes as revealed by domain-specific antibodies. J Biol Chem 273:17832-17838

26. Pikas DS, Li JP, Vlodavsky I, Lindahl U (1998) Substrate specificity of heparanases from human hepatoma and platelets. J Biol Chem 273:18770-18777

27. van den Hoven MJ, Wijnhoven TJ, Li J-P et al (2007) Reduction of anionic sites in the glomerular basement membrane by heparanase does not lead to proteinuria. Kidney Int (in press)

28. Lensen JF, Rops AL, Wijnhoven TJ et al (2005) Localization and functional characterization of glycosaminoglycan domains in the normal human kidney as revealed by phage display-derived single chain antibodies. J Am Soc Nephrol 16:1279-1288

29. Onozaki A, Midorikawa S, Sanada H et al (2004) Rapid change of glucose concentration promotes mesangial cell proliferation via VEGF: inhibitory effects of thiazolidinedione. Biochem Biophys Res Commun 317:24-29

30. Levidiotis V, Kanellis J, Ierino FL, Power DA (2001) Increased expression of heparanase in puromycin aminonucleoside nephrosis. Kidney Int 60:1287-1296 
31. Levidiotis V, Freeman C, Tikellis C, Cooper ME, Power DA (2004) Heparanase is involved in the pathogenesis of proteinuria as a result of glomerulonephritis. J Am Soc Nephrol 15:68-78

32. Levidiotis V, Freeman C, Tikellis C, Cooper ME, Power DA (2005) Heparanase inhibition reduces proteinuria in a model of accelerated anti-glomerular basement membrane antibody disease. Nephrology (Carlton) 10:167-173

33. Kramer A, van den Hoven M, Rops A et al (2006) Induction of glomerular heparanase expression in rats with adriamycin nephropathy is regulated by reactive oxygen species and the reninangiotensin system. J Am Soc Nephrol 17:2513-2520

34. Wijnhoven TJ, Geelen JM, Bakker M et al (2007) Adult and paediatric patients with minimal change nephrotic syndrome show no major alterations in glomerular expression of sulphated heparan sulphate domains. Nephrol Dial Transplant 22:28862893

35. Wijnhoven TJ, Lensen JF, Wismans RG et al (2007) In vivo degradation of heparan sulfates in the glomerular basement membrane does not result in proteinuria. J Am Soc Nephrol 18:823-832

36. Harvey SJ, Jarad G, Cunningham J et al (2007) Disruption of glomerular basement membrane charge through podocyte-specific mutation of agrin does not alter glomerular permselectivity. Am J Pathol 171:139-152

37. Chen S, Holzman L, Yamaguchi Y, Woods A, McCarthy DJ, McCarthy KJ (2006) Elimination of heparan sulfate glycosaminoglycans (HS-GAG) from the glomerular basement membrane (GBM) using Cre-Lox technology has profound effects on podocyte ultrastructure and function. J Am Soc Nephrol 17:25A

38. Morita H, Yoshimura A, Inui K et al (2005) Heparan sulfate of perlecan is involved in glomerular filtration. J Am Soc Nephrol 16:1703-1710
39. Myrup B, Hansen PM, Jensen T et al (1995) Effect of low-dose heparin on urinary albumin excretion in insulin-dependent diabetes mellitus. Lancet 345:421-422

40. Szelachowska M, Poplawska A, Topolska J et al (1997) A pilot study of the effect of the glycosaminoglycan sulodexide on microalbuminuria in type I diabetic patients. Curr Med Res Opin 13:539-545

41. Skrha J, Perusicova J, Pont'uch P, Oksa A (1997) Glycosaminoglycan sulodexide decreases albuminuria in diabetic patients. Diabetes Res Clin Pract 38:25-31

42. Dedov I, Shestakova M, Vorontzov A, Palazzini E (1997) A randomized, controlled study of sulodexide therapy for the treatment of diabetic nephropathy. Nephrol Dial Transplant 12:2295-2300

43. Gambaro G, Kinalska I, Oksa A et al (2002) Oral sulodexide reduces albuminuria in microalbuminuric and macroalbuminuric type 1 and type 2 diabetic patients: the Di.N.A.S. randomized trial. J Am Soc Nephrol 13:1615-1625

44. Tamsma JT, van der Woude FJ, Lemkes HH (1996) Effect of sulphated glycosaminoglycans on albuminuria in patients with overt diabetic (type 1) nephropathy. Nephrol Dial Transplant 11:182-185

45. van der Pijl JW, van der Woude FJ, Geelhoed-Duijvestijn PH et al (1997) Danaparoid sodium lowers proteinuria in diabetic nephropathy. J Am Soc Nephrol 8:456-462

46. Naggi A, Casu B, Perez M et al (2005) Modulation of the heparanase-inhibiting activity of heparin through selective desulfation, graded $\mathrm{N}$-acetylation, and glycol splitting. J Biol Chem 280:12103-12113

47. Kumar N, Bentolila A, Domb AJ (2005) Structure and biological activity of heparinoid. Mini Rev Med Chem 5:441-447

48. Levidiotis V, Freeman C, Punler M et al (2004) A synthetic heparanase inhibitor reduces proteinuria in passive Heymann nephritis. J Am Soc Nephrol 15:2882-2892 